\title{
Le jeu vidéo médiateur de savoirs en histoire : l'exemple de Crusader King 2 et Europa Universalis 4
}

The video game as mediator of knowledge in history: the example of Crusader Kings 2 and Europa Universalis 4

\section{Clément Dussarps}

\section{(2) OpenEdition}

\section{Journals}

Édition électronique

URL : http://journals.openedition.org/sdj/2696

DOI : $10.4000 /$ sdj.2696

ISSN : 2269-2657

\section{Éditeur}

Laboratoire EXPERICE - Centre de Recherche Interuniversitaire Expérience Ressources Culturelles Education

Référence électronique

Clément Dussarps, "Le jeu vidéo médiateur de savoirs en histoire : l'exemple de Crusader King 2 et Europa Universalis $4 »$, Sciences du jeu [En ligne], $13 \mid 2020$, mis en ligne le 12 novembre 2020, consulté le 21 janvier 2021. URL : http://journals.openedition.org/sdj/2696 ; DOI : https://doi.org/10.4000/sdj. 2696

Ce document a été généré automatiquement le 21 janvier 2021.

\section{(i) $\$$

La revue Sciences du jeu est mise à disposition selon les termes de la Licence Creative Commons Attribution - Pas d'Utilisation Commerciale - Pas de Modification 4.0 International. 


\title{
Le jeu vidéo médiateur de savoirs en histoire : l'exemple de Crusader King 2 et Europa Universalis 4
}

The video game as mediator of knowledge in history : the example of Crusader Kings 2 and Europa Universalis 4

\author{
Clément Dussarps
}

1 Le jeu vidéo est « un objet de réflexion, d'analyse et un outil de travail » (Minassian et Rufat, 2008), qui mérite d'être étudié en tant qu'objet de recherche, au moins pour deux raisons : la pratique du jeu vidéo se généralise à tous les âges et sexes, en particulier chez les adolescents (Médiamétrie, 2019) et l'industrie vidéoludique est devenue la première industrie culturelle (en termes de vente) en France (Lafrance et al., 2012, p. 9 ; Poinsignon, 2018) et dans le monde (Duris, 2017, p. 86). Une « véritable culture vidéoludique s'est constituée » (Amato, 2014, p. 164). Certains jeux se veulent de plus en plus réalistes, y compris lorsqu'ils proposent de vivre certaines époques avec un désir de fidélité plus ou moins abouti. En résulte ainsi un jeu qui, en plus d'être un média à la pratique ludique, se constitue comme une potentielle source d'apprentissages, notamment sur le plan historique. Nous souhaitons alors montrer en quoi deux jeux vidéo (sélectionnés par nos soins) peuvent être médiateurs d'apprentissages à deux niveaux : 1) par le jeu lui-même, et 2) par les recherches effectuées grâce à la curiosité suscitée par la pratique du jeu.

2 Après avoir rappelé que le jeu vidéo est un média et qu'il peut être un support de savoirs, nous présenterons la méthodologie mise en œuvre pour répondre à nos questions (une enquête en ligne) ainsi que les deux jeux étudiés dans cet article. Les résultats permettront de répondre à nos questions et d'ouvrir de nouvelles pistes de recherche. 


\section{Ancrage théorique}

\section{Le jeu vidéo : un média spécifique}

3 Le jeu vidéo est complexe à définir, tant ses origines sont plurielles : il est " pris dans une histoire du jeu et de l'enfance, mais aussi dans une évolution générale du capitalisme et des industries culturelles : le cinéma [...], la littérature jeunesse, les dessins animés, la bande dessinée, la fantasy, la science-fiction... et surtout le jouet » (Berry, 2012, p. 34). Il est un objet à la fois «social, culturel et technique unique » (Zabban, 2012, p. 176), ou encore "média cybernétique de masse» (Amato, 2014). D'autres auteurs présentent le jeu vidéo comme un média: il est un support de diffusion de l'information (Schafer et Thierry, 2012), une «technologie moderne de l'information et de la communication » (Bélisle et al., 1999, p. 200-201). Nous retrouvons ici les mêmes critères qui font de la bande dessinée un média selon Dacheux (2009, p.12): c'est un «support de communication aux caractéristiques techniques spécifiques » (qui existe désormais sur les principales technologies numériques du quotidien), "une industrie culturelle particulière » (les jeux vidéo sont d'ailleurs devenus le bien culturel le plus vendu en France selon Lafrance et al., 2012, p. 9), et "une médiation spécifique entre un public et des auteurs ", caractéristique qui nous intéressera particulièrement dans cet article.

4 Toutefois, le jeu vidéo se différencie d'autres médias comme le cinéma ou la bande dessinée, comme l'explique Genvo (2011, p. 96), car s'il " procède d'un acte narratif », le joueur est invité à agir et c'est lui qui guide les événements, bien qu'il soit contraint par un cadre plus ou moins restrictif, de par une trame scénaristique préétablie et organisée d'une certaine manière (la conception de niveau de jeu ou level design), et sa propre pratique du jeu. Ces éléments de scénarisation sont importants dans le cadre de notre étude, car nous analyserons des jeux dits "bacs à sable ", où le joueur jouit d'une grande liberté et où chaque partie est susceptible d'être très différente d'une autre, les objectifs étant propres aux joueurs ; Borzakian (2013) parle de « quasi-jeu ».

\section{« Jouer pour apprendre : est-ce bien sérieux » ?}

Le jeu peut être considéré comme "dénué de tout intérêt matériel et de toute utilité " (Huizinga, 1951, p. 31), dimension que l'on retrouve dans les discours sur les jeux vidéo (Lafrance et al., 2012), ou inversement comme « favorisant l'apprentissage, la détente, la régénération de soi et l'évasion» (Amato, 2012, p. 22), ayant des fonctions sociocognitives et sémiotiques (Perriault, 2012) qui nécessitent certains apprentissages afin de progresser. Caillois, bien que le définissant comme improductif sur un plan économique (p. 43), voit des qualités pour l'individu qui joue : «chaque jeu renforce, aiguise quelque pouvoir physique ou intellectuel» (Caillois, [1958]1967, p. 21). En tant que produit industriel, le jeu est susceptible d'être à la fois produit de divertissement et d'apprentissage : il « quitte la sphère du frivole, du pur divertissement, pour devenir sérieux, apparaître comme un support d'éducation spontanée " (Brougère, 2003, p. 44-45). Si nous définissons le jeu comme « un système basé sur des règles avec des résultats variables et mesurables, dans lequel ces résultats obtiennent différentes valeurs, et au sein duquel le joueur fait des efforts pour influencer ces résultats et se sent attaché émotionnellement à ceux-ci [...]» (Juul, [2005] 2011, p. 36, notre 
traduction), alors l'action de jouer implique cognitivement et affectivement le joueur ; ces dimensions sont importantes dans l'acte d'apprendre (Trocmé-Fabre, 1999).

6 Même si la raison première de pratiquer un jeu semble être de l'ordre du loisir, il peut être vecteur d'apprentissages. La particularité du jeu, lorsqu'il est un support d'apprentissage, est de mêler plaisir (dimension ludique) et effort (nécessaire à l'apprentissage), comme le souligne Giordan (interviewé par Darras, 2003), du moins si l'on désire réellement jouer avec. Mais ce couple ne se suffit pas à lui-même : si l'on fait des efforts pour réussir dans le jeu parce qu'on en retire du plaisir, on n'apprend pas nécessairement avec. De plus, Berry (2006) explique :

nous savons aujourd'hui que non seulement le jeu n'est pas une activité purement «biologique » ou naturelle pour apprendre, mais qu'elle est socialement construite (Bruner, 1987). Ainsi, la relation entre jeu et apprentissage est une relation peu évidente et elle relève plus souvent d'une pensée romantique (Brougère, 1995), d'un argument marketing (Kline, 2003), d'une construction sociale, d'une rhétorique (Sutton-Smith, 1997) que d'une réalité empirique.

Sa critique ne relève pas d'une vision «anti » jeux pour apprendre: il rappelle simplement que le jeu est un potentiel support d'apprentissage. "Il se trouve qu'en jouant il se peut qu'on apprenne, et qu'en apprenant on se trouve face à des situations qui ressemblent de près ou de loin au jeu » (Brougère, 2005, p. 166) ; l'apprentissage par le jeu est donc loin d'être évident. Berry fait la même observation avec les jeux sérieux (Berry, 2011).

8 La distinction entre jeu vidéo et jeux sérieux n'est pas toujours claire. Puisque le premier est un potentiel vecteur d'apprentissage (Berry, 2011) et que le second est par définition un jeu dédié à des apprentissages, la différence entre « jeux sérieux » et « jeu vidéo " pourrait tenir aux intentions de conception (usages prévus par les concepteurs, plutôt pour se divertir ou plutôt pour apprendre) et surtout aux pratiques (du point de vue des usagers). Un jeu sérieux pourrait être perçu et utilisé dans sa fonction ludique par certaines personnes, et inversement: un objet n'est en soi pas éducatif, c'est l'activité qui est faite avec qui peut l'être, y compris lorsqu'on joue (Brougère, 2019). De plus, nous sommes personnellement critique quant aux jeux « ludo-éducatifs » car, trop souvent, la séparation entre les connaissances et la dimension ludique est importante dans la pratique du jeu. Par exemple, le jeu Soldats inconnus : Mémoires de la Grande Guerre (Ubisoft, 2014) dédié à l'apprentissage de l'histoire apparaît décevantcar les connaissances ont une place anecdotique, et l'histoire racontée ne rend que très peu compte de faits historiques. Pour trouver des "morceaux d'histoire», le joueur doit, parfois péniblement, trouver des objets (inutiles pour la progression dans le jeu) et lire le texte associé ; la dimension ludique ne sert pas de support à l'apprentissage car il en est séparé. Dans Assassin's Creed (série développée par Ubisoft depuis 2007) ou Civilization (série développée par MicroProse de 1991 à 1999, Firaxis depuis 2000), nous retrouvons ce même défaut : des savoirs sont accessibles, mais nettement isolés du jeu ; il n'y a, par exemple, aucun lien avec les mécaniques et règles du jeu, ni le besoin d'y chercher quelque chose qui permettrait d'obtenir un score plus élevé ou un quelconque élément dans le jeu. 


\section{Le jeu vidéo : médiateur de savoirs?}

\section{Apprentissages non-disciplinaires avec les jeux vidéo}

Berry (2011) relie la pratique du jeu vidéo à l'apprentissage informel, soit un apprentissage similaire à celui que l'on peut avoir au sein des communautés de pratiques (Wenger, 1998), en particulier, dans son exemple, des jeux de rôles en ligne massivement multijoueurs. Il met en avant les éléments sociaux et anthropologiques, dans lesquels s'inscrivent les joueurs, comme sources d'apprentissages informels. Il définit un ensemble d'apprentissages possibles par le jeu (vidéo notamment) :1) tout ce qui a trait à la mémoire, l'attention visuelle, la représentation iconique, la manipulation et la représentation d'espaces en 3D ; 2) la coordination «main-œil », la dextérité et la rapidité d'analyse visuelle (notamment en mouvement), caractéristiques des jeux de tir ; 3) la compétence pour le multitâche (présence distribuée ou attention divisée) ; 4) la connaissance des règles du jeu et le fait d'établir des stratégies en fonction de ces règles, mais également la connaissance de la programmation du jeu (et donc aussi de ses failles) ; 5) la capacité à penser globalement et en hypertexte (il cite à ce sujet Gee, 2009), c'est-à-dire que les structures cognitives seraient alors parallèles et non séquentielles ; enfin, 6) des apprentissages liés non pas au jeu en lui-même, mais à la dimension communautaire autour du jeu (capacité à programmer, créer des "mods" pour les jeux, des vidéos, une narration, etc.). L'aspect communautaire est aussi un ensemble de compétences plus ou moins transférables dans la vie personnelle et professionnelle : «entraide, écoute des autres, apprentissage de la gestion de conflits au sein d'un même groupe " (Berry, 2011). À cela nous pouvons ajouter la capacité de travailler en groupe, à solliciter des personnes-ressources sur des éléments précis. En somme, une forme d'autonomie et d'organisation où l'on sait identifier et appeler les personnes-ressources en cas de besoin, voire diriger/organiser un groupe. Le jeu vidéo permettrait alors de développer des savoirs et/ou compétences sociales, sensorimotrices (Sudnow, 1983) et cognitives: mémoire, attention, représentation spatiale (Calvert, 2005) et de résolution de problèmes (Gee, 2005). Les compétences sociales seraient bien entendu plus à même d'être développées par des pratiques en multijoueurs.

\section{Apprentissages disciplinaires avec les jeux vidéo : quelques exemples}

Qu'en est-il des savoirs disciplinaires? Le sujet est moins souvent abordé dans la littérature scientifique lorsqu'il ne s'agit pas de jeux sérieux (dont le contenu est préchoisi pour être un outil d'apprentissages disciplinaires ou procéduraux/ méthodologiques), même si des discours institutionnels l'abordent ${ }^{2}$; les publics adultes font également moins souvent l'objet d'études à ce sujet.

11 Pour cela, nous allons d'abord préciser ce que nous entendons par les notions de donnée, d'information, de connaissance et de savoir. La donnée est un élément brut (une valeur, une mesure, une chaîne de caractère) dénué de tout contexte, de tout sens. L'information est la mise en relation des données entre elles (Balmisse, 2002), une contextualisation, pour leur donner du sens, résultant d'un effort cognitif de l'individu qui reçoit les données. La connaissance indique « le travail productif des sujets sur euxmêmes pour s'approprier des idées ou des méthodes ", celui de savoir " [caractérise] les formes de connaissance qui sont reconnues par une société » (Jeanneret, 2000) ; c'est 
pourquoi nous emploierons l'expression de «savoirs disciplinaires ", désignant dans cet article des savoirs en histoire et/ou géographie. Ainsi, le jeu vidéo est un support d'information et ces informations sont susceptibles de devenir des connaissances et savoirs.

Koebel (2018) montre que le jeu vidéo peut être un très bon support pour apprendre des concepts, comme se représenter des périodes historiques et prendre conscience des divergences de point de vue entre historiens, favorisant alors le développement d'un esprit critique à ce sujet. Minassian et Rufat (2008) exposent également l'intérêt de jeux de construction de villes utilisés comme support pédagogique :

Les jeux vidéo [...] assument, dans une certaine mesure, un rôle pédagogique par la pratique de l'espace, celle des représentations et des liens entre cet espace et les sociétés qui le produisent. Ensuite, les simulations produites par les jeux vidéo peuvent être des outils de modélisation [...]. Ils pourraient servir à expérimenter la modélisation de jeux d'acteurs ou les simulations d'aménagement du territoire. [...] Enfin, l'espace virtuel à la fois support et produit des jeux vidéo est un objet géographique qui s'apparente au territoire.

Karsenti et al. (2019) indiquent que la pratique du jeu, lorsqu'elle est fortement immersive comme dans la série des Assassin's Creed (série de jeux d'action se déroulant à différentes époques et reproduisant parfois avec une grande fidélité les décors, développée par Ubisoft), "peut très aisément impliquer le joueur et amener sa concentration au plus haut afin de l'amener vers des apprentissages, tout en prenant du plaisir à jouer » (p. 5). Enfin, Mozelius et al. (2017), dans une étude basée sur trois jeux vidéo effectuée auprès de 635 joueurs d'environ 21 ans, mettent en lumière que l'apprentissage tangentiel (c'est-à-dire suite à des recherches effectuées pendant ou après la pratique du jeu) constitue une part importante des apprentissages, en plus de ceux directement effectués dans le jeu, particulièrement avec des jeux historiques (Crusader Kings et Civilization dans leur étude). Ils montrent que les jeux intégrant des contenus d'apprentissage dans la mécanique du jeu sont les plus appropriés pour l'apprentissage; plus les savoirs sont fusionnés avec le scénario et/ou les actions à mettre en œuvre par le joueur, meilleur sera son apprentissage, sans que, pour autant, il s'en rende compte.

\section{Questionnements et méthodologie mise en œuvre}

\section{Questions de recherche}

14 Nous nous demandons dans cette contribution en quoi les jeux vidéo peuvent être des médiateurs informels de savoirs. Par médiateurs, nous entendons cette fonction de médiation, "[de] sens et [de] signification au sens large ", assurée par tout média (Peraya, 1999). Nous émettons deux hypothèses: 1) la pratique de ces jeux permet d'accéder à des savoirs dans la mesure où ils en sont porteurs et, 2) cette pratique conduit les joueurs à aller chercher des informations sur différents supports au sujet d'informations rencontrées dans le jeu (source d'apprentissage tangentiel). Cette forme de médiation viendrait combler un besoin d'information, au sens où l'entend Simonnot (2007, p.41), «[portant] l'individu à s'engager dans une activité de recherche d'information", stimulant sa curiosité et le motivant dans cette voie; en somme, l'activité ludique crée-t-elle un besoin d'information et, si oui, comment et jusqu'à quel point l'usager comble-t-il ce besoin? 


\section{Les jeux étudiés} Europa Universalis 4 (EU4). Ces jeux sont conçus et édités par l'entreprise suédoise Paradox interactive et sortis respectivement en 2012 et 2013, faisant suite aux volets précédents. Si les dates de sortie paraissent aujourd'hui lointaines, des mises à jour et des extensions $\left(\mathrm{DLC}^{3}\right)$ sont proposées régulièrement (les dernières extensions sont parues en 2018 pour CK2 et 2020 pour EU4). Le nombre de joueurs actifs de ces jeux reste stable et, sur la plateforme Steam, ils restent parmi les 100 jeux les plus joués au moment de la rédaction de cet article ${ }^{4}\left(\mathrm{EU} 4\right.$ est $42^{\mathrm{e}}$ et $\mathrm{CK} 2{ }_{76^{\mathrm{e}}}$ ). Il est difficile de dire si le public est le même depuis leur sortie ou s'il se renouvelle régulièrement, mais ils sont loin d'être oubliés plusieurs années après leur sortie, comme en témoigne la communauté de joueurs toujours très active. extermination » ou «eXplore, eXpand, eXploit and eXterminate » en anglais), où le joueur incarne un personnage dans $\mathrm{CK} 2$ et une nation dans EU4. Ils ont comme trame de fond une histoire et une géographie fidèles aux connaissances actuelles ( $\mathrm{du}$ moins au démarrage d'une partie), détaillant avec précision les frontières et personnages en jeu. La date de départ peut être ajustée sur les deux jeux, y compris au jour près : l'écran de démarrage d'une nouvelle partie permet ainsi de modifier la date de départ et de voir les évolutions de frontières et/ou de personnages au cours du temps. Dans les deux cas, le joueur est amené à gérer un domaine plus ou moins vaste, sur les plans économiques, urbains, militaires et technologiques, à forger des alliances, déclencher des guerres ou se défendre, négocier une paix, gérer les relations religieuses, partir en croisade, gérer sa succession, etc. Les joueurs ont pour cela une interface complexe et de nombreuses fonctionnalités à disposition. Des événements, historiquement fidèles ou non, viennent ponctuer leurs parties (par exemple, l'union ibérique dans EU4). Le commencement des jeux se veut donc historiquement fidèle, mais la suite peut ne plus l'être du tout, le jeu laissant énormément de libertés et l'intelligence artificielle dirigeant les autres personnages ou nations ayant également des façons de procéder anhistoriques. En cela, c'est un jeu "bac à sable » : le joueur et l'ordinateur vont façonner un monde différent à chaque nouvelle partie. Il est possible de jouer en réseau dans les deux jeux, donnant lieu à des sessions plus ou moins longues. Enfin, même si le joueur enregistre un score qui est mis en comparaison avec les autres joueurs et/ou intelligences artificielles, il n'y a pas de réelle possibilité de gagner: la partie s'achève lorsque la date finale (prévue dans le jeu) est arrivée. À la lecture des forums, on constate que les joueurs se donnent souvent des objectifs de partie (parfois orientés par des éléments prévus par le jeu) dont l'atteinte met le plus souvent fin à la partie.

Les deux jeux nécessitent néanmoins d'être présentés séparément. CK2 couvre le Moyen-Âge, de $769^{5}$ (peu après le début des règnes de Charlemagne et Carloman I ${ }^{\text {er }}$ et avant les premières invasions vikings en Angleterre) à 1453 (chute de Constantinople et de l'Empire Romain d'Orient). On y dirige une dynastie (cf. figure 1 , celle de Charlemagne). L'accent est mis sur la gestion intérieure de son domaine (comté, duché, royaume ou empire), la relation avec les autres personnages (en particulier les vassaux) et la succession (notre personnage étant amené à mourir, plus ou moins rapidement selon divers événements). Le joueur peut se marier, avoir des enfants, comploter, faire la guerre, mettre en place un anti-pape, etc. Le jeu permet de jouer des nobles de 
royaumes chrétiens féodaux, et, avec certains DLC, d'incarner des personnages vikings, païens, arabo-musulmans, etc. À la différence d'EU4, le jeu limite la carte du monde à l'Europe, le nord de l'Afrique et ne couvre pas entièrement l'Asie. Selon le personnage joué, il accorde ainsi une place importante au système féodal. La gestion des vassaux, en particulier, peut constituer une grande partie du temps de jeu. CK2 a une forte dimension type "jeux de rôles ", proposant au joueur des événements le conduisant à choisir s'il veut diriger un personnage gentil, cynique, pieux, cruel, tyrannique, dépressif, etc. Il peut aussi tomber malade, être inapte à diriger, etc. Enfin, le jeu rend compte de différents événements avec une certaine précision au niveau des dates: première croisade, invasions vikings, conquête de l'Angleterre par Guillaume le Conquérant, invasions mongoles, etc.

Figure 1. Crusader Kings 2.

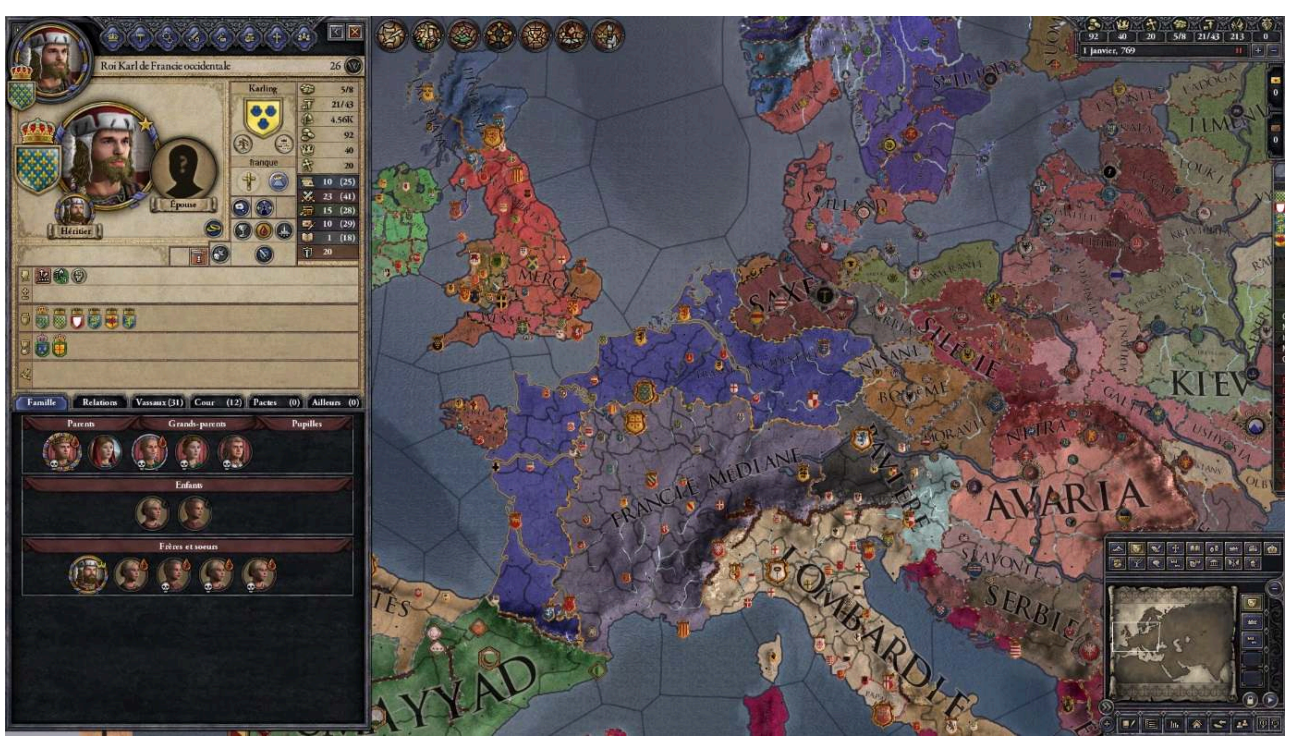

Interface montrant le personnage contrôlé et une partie du plateau de jeu (carte).

EU4 propose une date de départ en 1444 (jour de la bataille de Varna) et couvre la fin du Bas-Moyen-Âge, la Renaissance et les temps modernes jusqu'en 1821. Le joueur y dirige une nation. Malgré des points communs avec $\mathrm{CK} 2$, il s'agit surtout de faire prospérer son territoire, qui ici est divisé en provinces; le joueur n'a pas affaire à des vassaux comme dans CK2. Ainsi, la gestion est moins interne qu'externe, mettant l'accent sur la politique étrangère. On peut forger des alliances, faire la guerre, se développer sur les plans économique, militaire et technologique, et coloniser de nouveaux territoires (notamment l'Amérique), participer aux guerres de religions, développer de grandes routes commerciales, abolir l'esclavage, etc. Le jeu propose un système d'objectifs à remplir qui le différencie beaucoup de CK2 (qui n'en propose que rarement) : le joueur peut, certes, choisir de ne pas les suivre, mais il y est encouragé du fait des avantages que cela procure (surtout depuis diverses mises à jour qui, pour les nations jugées les plus importantes, propose une véritable trame à suivre, souvent liée à l'histoire ou à ce qu'elle aurait pu être) : selon la culture, religion et/ou nation jouée, on peut créer la Russie, la Prusse, l'Espagne, devenir Empereur du Japon, fonder les États-Unis d'Amérique, etc. Cela ne limite toutefois que peu la dimension «bac-à-sable » du jeu, puisque le joueur jouit toujours d'une très grande liberté. 

Caillois (1967), du fait de leurs règles complexes, précises et importantes pour prendre plaisir à jouer. L'effort nécessaire pour pratiquer de tels jeux avec plaisir est important. La pratique du jeu relève elle-même d'un certain étayage: on apprend au fur et à mesure à faire des actions de plus en plus complexes au cours des différentes parties jouées, à prévoir ce qui pourrait se passer si l'on fait certains choix et donc à anticiper, etc. Les nombreux tutoriels en ligne, forums dédiés et le Wiki officiel sont des sources d'aide importantes. Ainsi, le joueur est amené à passer du temps non seulement sur le jeu lui-même, mais aussi pour une recherche d'aide. Ces deux jeux se placent également du côté du simulacre (mimicry), du fait de la dimension bac à sable qui se veut une simulation de la réalité, et de la compétition (agôn), dans le sens du dépassement de soi (ou « compétition avec soi-même ", Yonnet, 2004, cité par Borzakian, 2013), nécessitant $\mathrm{du}$ talent et un effort important ( $\mathrm{du}$ moins la première centaine d'heures ${ }^{6}$ ), ce qui s'exprime par certains joueurs dans les récits de parties ou messages échangés sur les forums en ligne.

Notons que ces deux jeux sont peu étudiés par la recherche scientifique (Coavoux et al. écrivent d'ailleurs que les recherches sur les jeux vidéo concernent rarement ce type de jeux), plus encore en ce qui concerne l'apprentissage possible en y jouant. Paradox interactive propose d'autres jeux « $4 \mathrm{X}$ » sur fond historique : la série des Hearts of Iron (seconde guerre mondiale), dont le premier est sorti en 2002 et le dernier en 2016, et Victoria (1836 à 1936), édité en 2003 pour le premier, 2010 pour le second. Nous avons choisi de ne pas nous attarder sur ces jeux, car Hearts of Iron est orienté sur l'aspect militaire et peu sur l'histoire, et Victoria n'est plus maintenu à jour.

\section{Savoirs disciplinaires dans ces deux jeux}

Le joueur peut accéder à des informations qui, s'il se les approprie, constituent des connaissances et de potentiels savoirs disciplinaires, enseignés à différents niveaux (scolaires et universitaires): les cartes du jeu au démarrage d'une partie, les personnages de l'époque (pour CK2), qui varient selon l'époque choisie. La carte de démarrage indique au joueur le contexte historique selon l'époque choisie ( $c f$. figures 2 et 3). Elle permet aussi de voir les différentes religions selon les provinces, le nom des régions du monde (ainsi que les territoires dits de jure dans $\mathrm{CK} 2$ ), etc. 
Figure 2. Carte de démarrage d'EU4 (frontières).

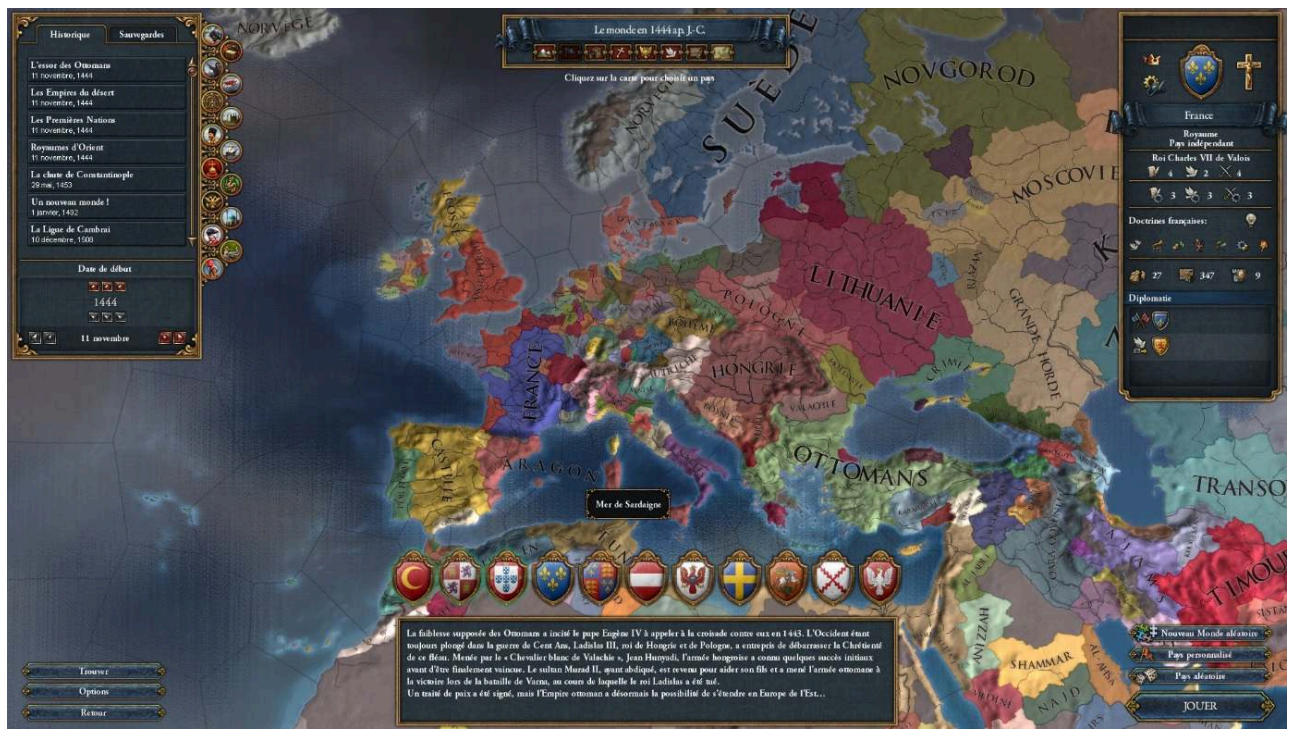

Le texte en bas indique le contexte de l'époque. Les drapeaux au-dessus indiquent les factions considérées comme les plus intéressantes à jouer à cette époque.

Figure 3. Carte de démarrage de EU4 (religions).

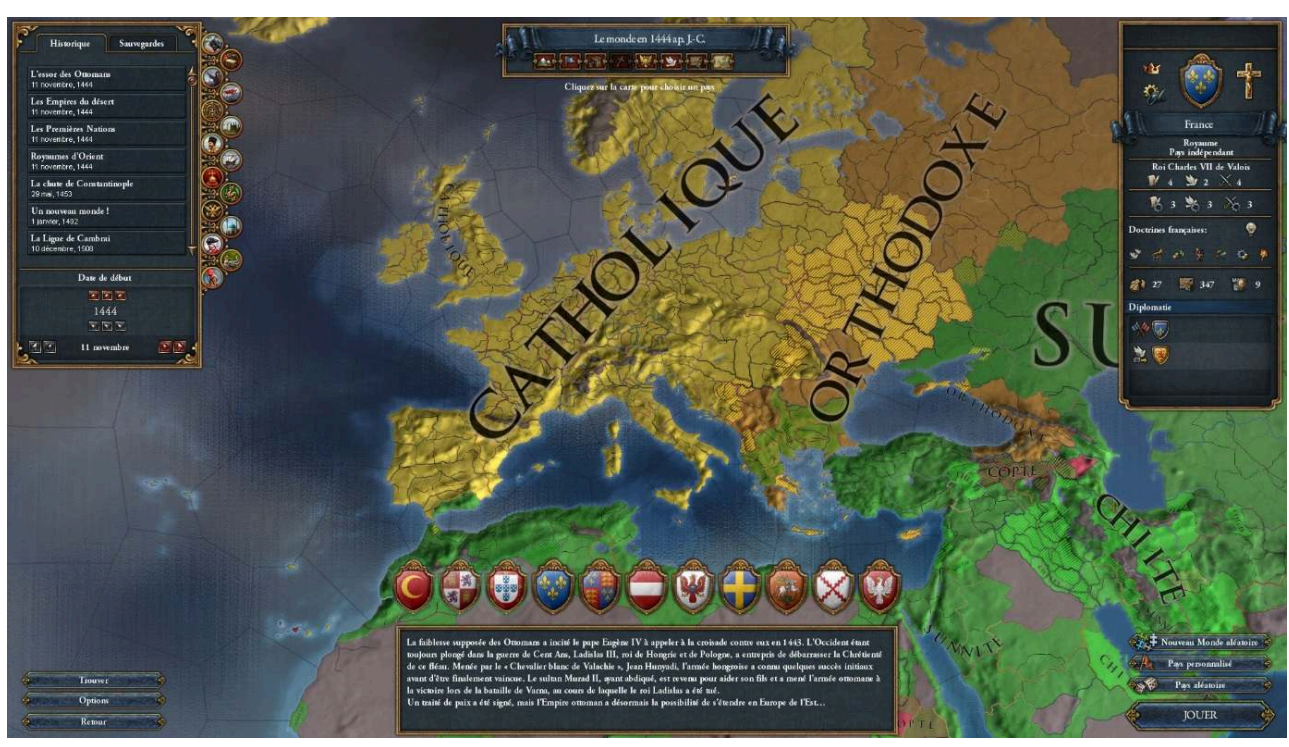

On peut également accéder directement à la page Wikipédia, adaptée à la langue du jeu, d'un personnage ayant existé dans CK2, qu'on l'incarne ou non (cf. figure 4). 
Figure 4. Lien Wikipédia vers le personnage

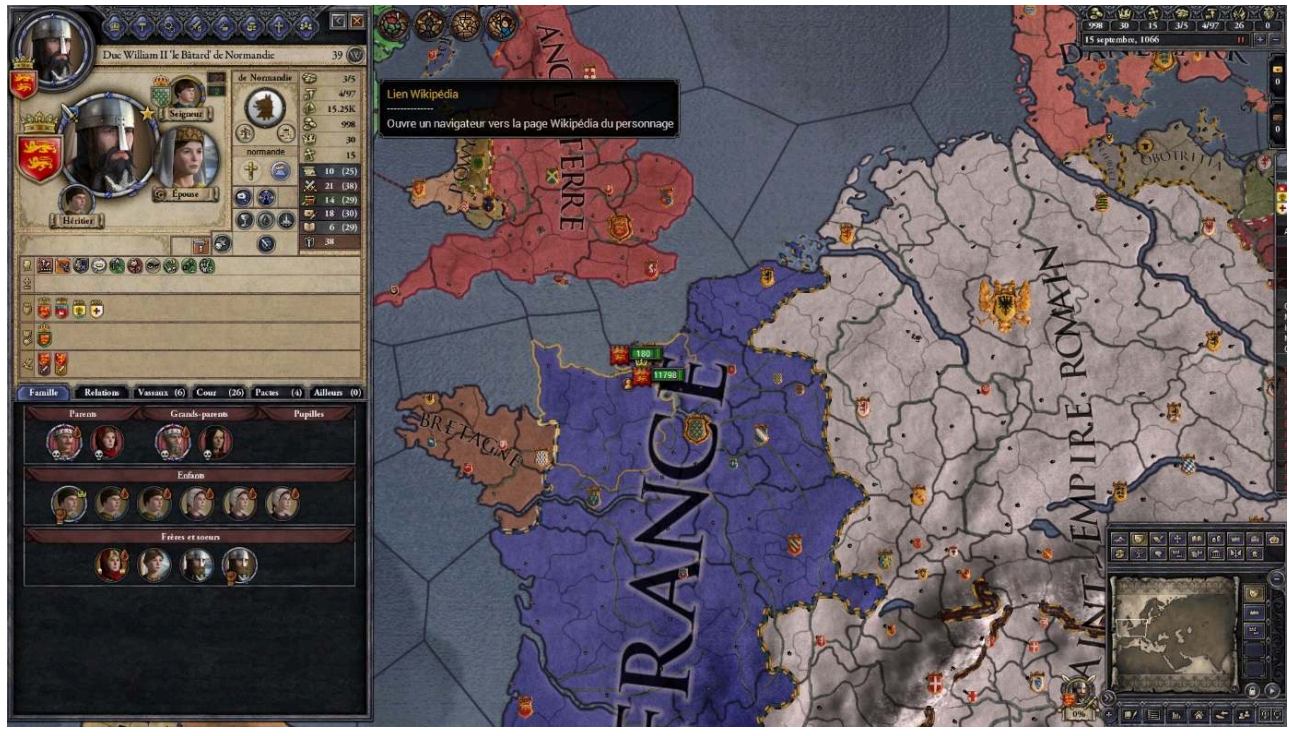

De plus, des infobulles permettent d'en savoir plus sur un système politique (par exemple, la féodalité), la religion ou encore la culture (cf. figures 5,6$)$.

Figure 5

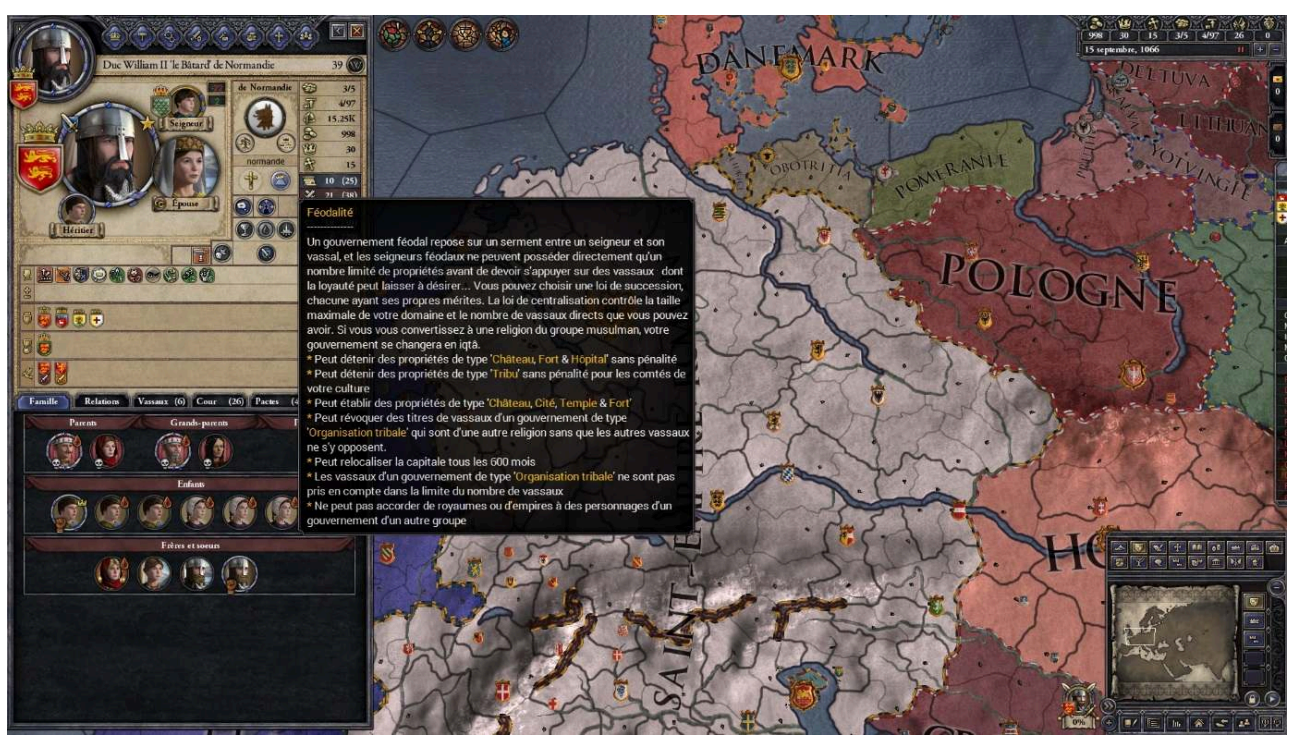

Infobulle sur la féodalité, contenant des informations historiques et d'autres liées uniquement au jeu 
Figure 6

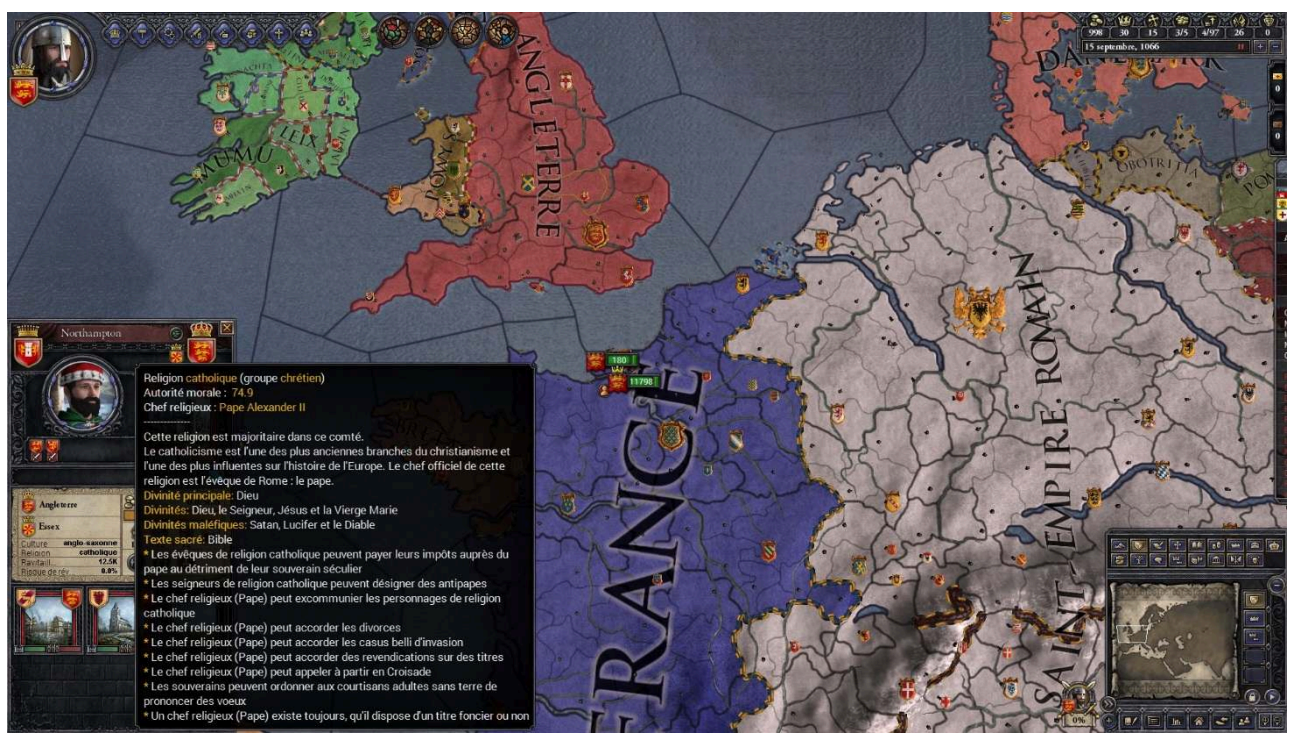

Infobulle sur la religion, contenant des informations historiques et d'autres liées uniquement au jeu

Dans CK2, l'on peut même avoir une idée plus ou moins précise de l'arbre généalogique d'un personnage (cf. figure 7).

Figure 7. Arbre généalogique (incomplet) de Guillaume le Conquérant.

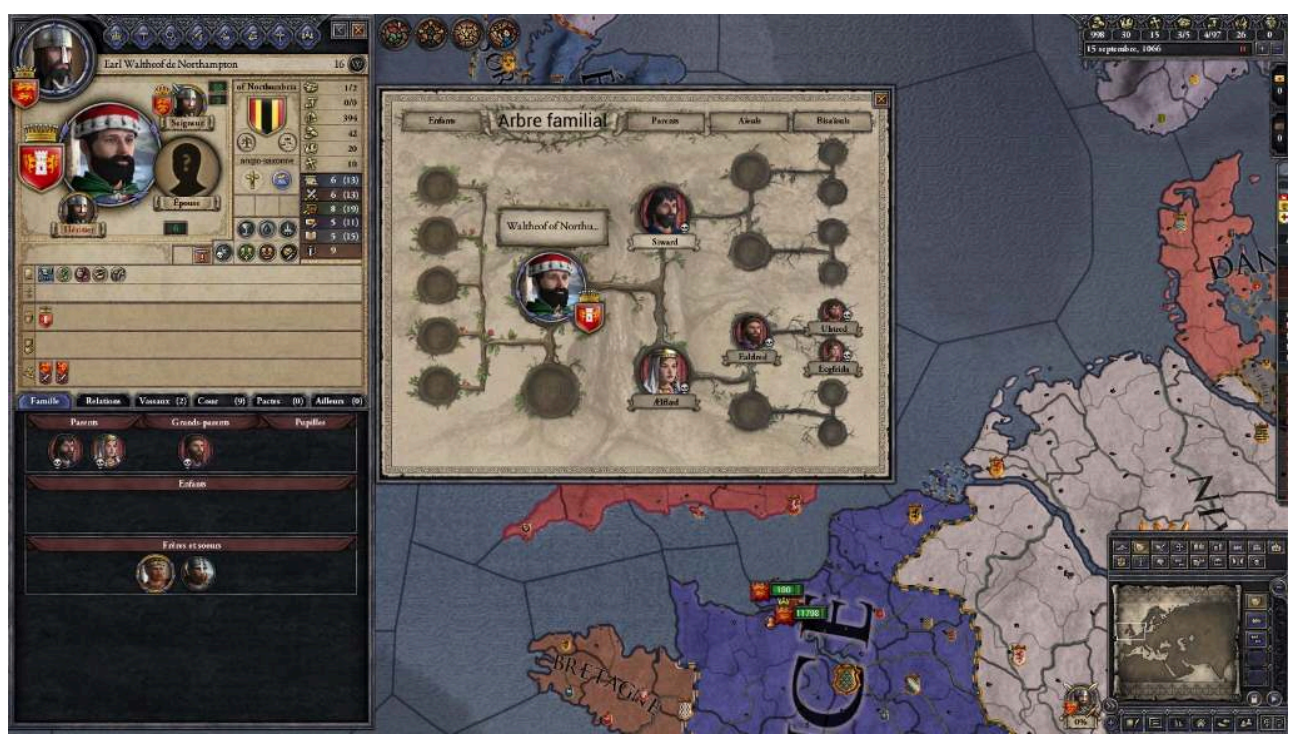

22 En outre, des événements historiques viennent ponctuer le jeu (plus souvent dans EU4, qui se veut plus fidèle à l'histoire), qui se déclencheront selon que les conditions soient ou non réunies (en fonction de ses actions et de celles de l'IA). Des infobulles permettent, ici encore, d'indiquer quelques éléments historiques au joueur.

\section{Enquête et échantillon}

Deux enquêtes par questionnaires ont eu lieu entre décembre 2017 et mars 2018 pour le premier, E1, (destiné aux joueurs d'EU4 et CK2), et avril et décembre 2018 pour le second, E2, (destiné à tout joueur de jeux vidéo, hors EU4 et CK2), dans un but d'abord 
exploratoire. Ce second questionnaire avait vocation à servir de comparaison entre des joueurs des deux jeux choisis et des joueurs d'autres jeux. Nous avons proposé d'y participer en écrivant un message sur divers forums : certains de jeuxvideo.com (sur les forums d'EU4 et CK2 pour le premier, sur 21 forums des jeux d'actualité à l'époque ${ }^{7}$ pour les autres, ainsi que le forum du «18-25 ans ») et de forum.reseau-js.com, forums d'un site web spécialisé dans les jeux de stratégie, en complément pour le premier questionnaire (à la même période). Ces sites web ont été sélectionnés 1) pour recueillir des réponses de joueurs français afin de cibler notre étude dans ce contexte particulier et 2) car ils sont très visités (le premier par des joueurs de tout jeu et le second par des joueurs de jeux de stratégie). Nous avons ainsi recueilli 570 réponses exploitables pour le premier questionnaire concernant EU4 et CK2 (E1), et 509 réponses pour le second (E2), dont 376 sont pleinement exploitables.

Les deux questionnaires comportaient cinq parties avec des questions identiques afin de faciliter la comparaison: le profil du joueur (notamment la fréquence d'achat de différents types de jeux, y a-t-il des jeux historiques parmi ceux-là, fréquence de jeux en général, en période de vacances et les week-ends, etc. $\left.{ }^{8}\right)$, le profil sociodémographique (sexe, âge, statut professionnel ou scolaire/étudiant, etc.), l'attrait pour l'histoire et pour la géographie, les apports d'EU et CK (tous numéros confondus) pour E1. Un jeu-questionnaire (quizz) suivait ( $c$ f. tableau 1), dans les deux questionnaires : il s'agit de sept questions liées à des connaissances en histoire et géographie sur la période concernée par $\mathrm{EU}$ et $\mathrm{CK}$; toutefois, la $4^{\mathrm{e}}$ question fait référence à un élément rarement évoqué dans EU (et jamais dans $\mathrm{CK}$ ) et la $7^{\mathrm{e}}$ n'est indiquée dans aucun des deux jeux. Il s'agissait dans ce quizz, construit de façon arbitraire afin de limiter le nombre de questions posées dans le questionnaire pour éviter la lassitude des répondants, de comparer les résultats entre $\mathrm{E} 1$ et $\mathrm{E} 2$, pour vérifier que les jeux étudiés sont bien source d'apprentissage (du moins pour les 7 questions posées). Une question, plus ouverte, leur demandait un exemple de ce qu'ils avaient appris dans ces jeux. Elle a recueilli 222 réponses ( $45 \%$ de l'échantillon E1).

Tableau 1. Questions du quizz et liens avec les deux jeux étudiés.

\begin{tabular}{|c|c|c|}
\hline $\mathrm{N}^{\circ}$ & Sujet concerné & Visible dans... \\
\hline Q1 & Saint Empire Romain Germanique & CK2 et EU4 \\
\hline Q2 & Expansion de l'Empire Ottoman (du XV $\mathrm{XV}^{\mathrm{e}}$ au XVIII ${ }^{\mathrm{e}}$ siècles) & $\begin{array}{l}\text { Partiellement dans } \\
\text { CK2 } \\
\text { EU4 }\end{array}$ \\
\hline Q3 & Réunion des royaumes de Castille et d'Aragon & EU4 \\
\hline Q4 & Vasco de Gama (trajet et découvertes) & $\begin{array}{l}\text { Rarement dans EU4 } \\
\text { (événement aléatoire) }\end{array}$ \\
\hline Q5 & $\begin{array}{l}\text { Division du duché de Bourgogne suite à la mort de Charles le } \\
\text { Téméraire }\end{array}$ & EU4 \\
\hline Q6 & Pologne-Lithuanie (République des deux nations) & EU4 \\
\hline
\end{tabular}


En complément, nous demandions aux joueurs où ils avaient appris neuf autres éléments (dans un jeu vidéo, en particulier dans EU/CK ou non, à l'école, dans un livre, dans un reportage, etc.). De la même manière, nous avons choisi d'être arbitraire quant au choix des questions, bien que, comme pour le quiz, certains éléments sont abordés dans les programmes scolaires. Ces éléments sont présentés par la suite dans le tableau 7. Les joueurs d'E1 pouvant être joueurs de CK2 uniquement, nous avons choisi de nous centrer sur des éléments européens. Une dernière question leur demandait s'ils acceptaient d'être contactés pour un entretien'.

Sur les 509 réponses à $\mathrm{E} 2,133$ se sont arrêtés à la partie " quizz », qui se trouvait avant la partie «profil socio-démographique » ce qui n'a permis pas d'exploiter ces réponses. Il est probable que les questions posées aient été trop difficiles pour des non-joueurs d'EU4 et de CK2 et les aient démotivés (voire frustrés) dans leur participation.

L'échantillon est donc opportuniste. Il est majoritairement masculin, âgé de 20 à 25 ans en moyenne, généralement d'un niveau d'études supérieur au baccalauréat (selon l'âge, certains sont collégiens ou lycéens) et soit scolarisé ou étudiant, soit en emploi pour la grande majorité ( $12 \%$ ont un autre statut dans l'E1, $16 \%$ dans l'E2, dont notamment des demandeurs d'emploi). Le tableau suivant décrit nos échantillons et permet de les comparer :

\section{Tableau 2}

\begin{tabular}{|l|l|l|l|l|l|l|l|l|}
\hline & \multicolumn{2}{|l|}{ Sexe (en \%) } & \multicolumn{2}{l|}{$\begin{array}{l}\text { Age (d'après l'année de } \\
\text { naissance) }\end{array}$} & \multicolumn{2}{l|}{ Statut ${ }^{10}$ (en \%) } \\
\hline & Hommes & Femmes & Moyenne & Médiane & $\begin{array}{l}\text { Ecart- } \\
\text { type }\end{array}$ & $\begin{array}{l}\text { Collégien ou } \\
\text { Lycéen }\end{array}$ & Etudiant & $\begin{array}{l}\text { En } \\
\text { emploi }\end{array}$ \\
\hline E1 & $98,5 \%$ & $1,5 \%$ & 28,2 ans & 23 ans & 6,82 & $19 \%$ & $38 \%$ & $31 \%$ \\
\hline E2 & $93,3 \%$ & $6,7 \%$ & 25,1 ans & 23 ans & 7,61 & $18 \%$ & $31 \%$ & $35 \%$ \\
\hline TOTAL & $96,5 \%$ & $3,5 \%$ & 26,9 ans & 23 ans & 7,16 & $19 \%$ & $35 \%$ & $32 \%$ \\
\hline
\end{tabular}

Profil socio-démographie et statut social des deux échantillons (E1 : 487 réponses, E2 : 318 réponses, du fait de non réponses à certaines questions).

28 Nos échantillons sont sensiblement différents des études sur le "profil du joueur français » : en 2014, $77 \%$ des hommes et $66 \%$ des femmes étaient des joueurs, et l'âge moyen du joueur était de $31 \mathrm{ans}^{11}$, alors qu'en 2019 la proportion était la même pour les hommes et les femmes $(68 \%)^{12}$ et l'âge moyen était de 39 ans $^{13}$. Toutefois, les études différencient rarement les joueurs sur téléphones mobiles, sur consoles et sur ordinateur (et notamment en croisant sexe et âge avec le support); or, dans notre étude, nous avons ciblé des jeux qui ne peuvent être joués que sur ordinateur (PC ou MAC) pour ce qui concerne l'enquête E1, ce qui peut expliquer certains écarts. En outre, les types de jeux ciblés (jeux de stratégie) semblent plus souvent pratiqués par des hommes ( $25 \%$ des joueuses contre $34 \%$ des joueurs $\left.{ }^{14}\right)$. 
Nous observons également quelques différences quant à la filière suivie au baccalauréat (actuellement ou précédemment) parmi les répondants concernés $(12 \%$ de nos répondants sont dans une classe inférieure à la terminale) : $90 \%$ d'E1 étaient (ou sont) dans une filière générale, contre $75 \%$ chez E2 ; les autres étaient (ou sont) dans une filière technologique $(8,1 \%$ chez E1 contre $25 \%$ chez E2) ou professionnelle $(1,6 \mathrm{chez}$ $\mathrm{E} 1$, aucun chez E2). Le tableau suivant montre la répartition des répondants selon leur niveau d'étude, parmi ceux ayant un baccalauréat ( $73 \%$ de notre échantillon) :

Tableau 3

\begin{tabular}{|l|l|l|l|l|l|}
\hline & $\begin{array}{l}\text { BAC ou équivalent } \\
\text { (en \%) }\end{array}$ & $\begin{array}{l}\text { BAC }+1 \\
+2 \\
(\mathrm{en} \%)\end{array}$ & $\begin{array}{l}\mathrm{BAC}+3 /+4 \\
(\mathrm{en} \%)\end{array}$ & $\begin{array}{l}\mathrm{BAC}+5 \text { et plus } \\
(\mathrm{en} \%)\end{array}$ & $\begin{array}{l}\text { TOTAL } \\
(\mathrm{en} \%)\end{array}$ \\
\hline E1 & 33 & 21 & 23 & 24 & 100 \\
\hline E2 & 29 & 23 & 25 & 23 & 100 \\
\hline TOTAL & 31 & 21 & 24 & 24 & 100 \\
\hline
\end{tabular}

Niveau d'études des répondants au niveau supérieur ou égal au baccalauréat. $N=584$.

Notons enfin qu'il y a une disparité entre les répondants à E1 et E2 concernant leur fréquence de jeu et les moments choisis pour jouer, en corrélation avec leur âge et leur statut social (toutefois, les étudiants sont également concernés par le fait de moins jouer en semaine et plus le week-end). Nous leur avons demandé leur fréquence de sessions de jeu et la durée approximative d'une session, comme le montre le tableau 4. Ainsi, les joueurs d'EU4 et CK2 sont plus souvent des joueurs « du week-end ", peut-être $\mathrm{du}$ fait d'une vie professionnelle et/ou étudiante plus chargée, ou encore de la dimension potentiellement chronophage des jeux auxquels ils jouent, bien qu'il soit possible de faire de courtes sessions sur ces derniers. La parentalité n'est pas un facteur discriminant (11\% des répondants d'E1 ont des enfants, $12 \%$ de ceux d'E2).

Tableau 4

\begin{tabular}{|l|l|l|l|}
\hline Fréquence et temps de session de jeu & $\begin{array}{l}\text { E1 } \\
\text { (en \%) }\end{array}$ & $\begin{array}{l}\text { E2 } \\
\text { (en \%) }\end{array}$ & $\begin{array}{l}\text { TOTAL } \\
\text { (E1 et E2, en \%) }\end{array}$ \\
\hline Joue tous les jours ou presque et plus de 5h à chaque fois & 15 & 24 & 19 \\
\hline Joue souvent, mais moins longtemps (2h à 5h) & 14 & 18 & 16 \\
\hline Joue souvent, mais peu de temps (1h ou moins) & 7 & 8 & 8 \\
\hline Joue essentiellement le week-end & 48 & 39 & 44 \\
\hline Joue essentiellement pendant les vacances & 4 & 2 & 3 \\
\hline
\end{tabular}




\begin{tabular}{|l|l|l|l|l|}
\hline Joue peu & 12 & 9 & 10 \\
\hline
\end{tabular}

Fréquence et temps de jeux selon les enquêtes (E1 : 570 réponses ; E2 : 490 réponses ; TOTAL $=1060$ )

\section{Résultats de l'enquête}

\section{Les joueurs d'EU et CK : des profils de joueurs spécifiques}

\section{Types de jeux joués}

31 Nous avons demandé dans l'enquête les types de jeux achetés et joués, et la fréquence d'achat. Nous constatons que les joueurs d'EU4 et CK2 jouent plus souvent à des jeux de stratégie et/ou de gestion (et $93 \%$ d'entre eux jouent en alternance à EU4 et/ou CK2 et d'autres jeux vidéo). Plus encore, ces joueurs semblent se spécialiser dans ce type de jeux : $71 \%$ jouent principalement à des jeux de stratégie (avec un contexte historique comme la série des Civilization, des Total War (The Creative Assembly, depuis 2000), ou encore des Age of Empires -Ensemble Studios, MacSoft Games et Zio Interactive, depuis 1997 ${ }^{15}$ ) ou de gestion (Sim City ${ }^{16}$ ou Cities Skylines ${ }^{17}$ notamment).

Tableau 5

\begin{tabular}{|l|l|l|l|}
\hline Joueurs de... (en \%) & E1 & E2 & E1 et E2 \\
\hline Tout type de jeux & 2 & 2 & 2 \\
\hline Tout type de jeux sauf jeux de sport & 9 & 8 & 9 \\
\hline Jeux de stratégie et/ou de gestion & 71 & 48 & 60 \\
\hline Autres types de jeux ${ }^{18}$ & 18 & 42 & 29 \\
\hline TOTAL & $\mathbf{1 0 0}$ & $\mathbf{1 0 0}$ & $\mathbf{1 0 0}$ \\
\hline
\end{tabular}

Types de jeux principalement joués par les différents enquêtés. N =1047. La question posée était «à quelle fréquence achetez-vous ou téléchargez-vous les types de jeux suivants ? » et les réponses possibles, en échelles sur différents types de jeux, « Jamais ; Rarement ; 2 à 3 fois par an ; 4 à 6 fois par an ; Plus souvent ». Les valeurs considérées dans le tableau concernent 2 fois par an ou plus.

Les joueurs d'E1 y jouent ou y ont joué avec globalement plus d'intensité que ceux d'E2, comme le montre le tableau suivant :

Tableau 6

\begin{tabular}{|c|c|c|c|c|c|c|c|c|c|c|}
\hline \multirow{3}{*}{ Jeu de la série... } & \multicolumn{10}{|c|}{ Temps de jeu estimé (en \%) } \\
\hline & \multicolumn{2}{|c|}{ Jamais } & \multicolumn{2}{|c|}{ Quelques heures } & \multicolumn{2}{|c|}{20 à 50 heures } & \multicolumn{2}{|c|}{50 à 150 heures } & \multicolumn{2}{|c|}{ + de 150 heures } \\
\hline & E1 & E2 & E1 & E2 & E1 & E2 & E1 & E2 & E1 & E2 \\
\hline Total War ${ }^{19}$ & 22 & 50 & 10 & 9 & 8 & 7 & 16 & 8 & 44 & 26 \\
\hline
\end{tabular}




\begin{tabular}{|c|c|c|c|c|c|c|c|c|c|c|}
\hline Civilization & 13 & 58 & 14 & 4 & 19 & 6 & 19 & 12 & 33 & 20 \\
\hline Age of Empires & 19 & 30 & 11 & 10 & 18 & 15 & 19 & 16 & 33 & 29 \\
\hline $\begin{array}{l}\text { Hearts of } \\
\text { Iron }\end{array}$ & 45 & 87 & 14 & 5 & 11 & 3 & 13 & 2 & 17 & 3 \\
\hline
\end{tabular}

Temps de jeu estimé par les répondants à E1 (488) et E2 (314) à quelques jeux de stratégie historiques. La lecture se fait en ligne (sur $100 \%$, par enquête). La question posée était : " Avez-vous joué aux jeux vidéo suivants, et combien de temps », avec les modalités de réponse suivantes pour chaque jeu : « Jamais ; Testé quelques heures; Joué entre 20 et 50 heures ; Joué entre 50 et 150 heures; Plus de 150 heures ».

Notre liste étant incomplète, un champ libre permettait de citer d'autres jeux historiques. Est citée le plus souvent sur E1 la série des Caesar (Golden Sector Design, 1992), où le joueur incarne le gouverneur d'une cité et doit développer la ville et satisfaire à un certain nombre de critères comme le bonheur des citoyens ou l'hygiène (à la manière de ce qui est appélé en anglais un city builder), gérer les infrastructures et, parfois, faire la guerre. Sur E2, les valeurs diffèrent : parmi les plus cités se trouvent Age of Mythology (un jeu de stratégie qui reprend les principes d'Age of Empires en se basant sur les mythologies égyptiennes, nordiques et grecques), Battlefield (DICE, depuis 2002) qui est un jeu de tir à la première personne qui s'est parfois basé sur des périodes historiques comme la Première ou la Seconde Guerre mondiale, Assassin's Creed qui, s'il est un jeu d'action, se base sur différentes périodes historiques, notamment pour la reproduction des villes et décors de l'époque.

Ceci nous éclaire sur la difficulté à identifier des jeux vidéo historiques (ou plutôt «à caractère historique »), tant pour des joueurs que les chercheurs sur le sujet, et à les classer par "profondeur historique »: Civilization est peu historique en apparence, mais le joueur peut y identifier les différentes ères (de la préhistoire à notre époque), les noms de quelques dirigeants et de leur peuple, les merveilles du Monde, etc., ou encore la série des Assassin's Creed rend compte d'une ambiance et d'une architecture d'époque, même s'il y a peu de faits historiques. Toutefois, nous constatons que peu de jeux nécessitant autant d'implication pour apprendre à jouer qu'EU4 et CK2 sont cités, révélant probablement une différence de publics entre $\mathrm{E} 1$ et $\mathrm{E} 2$, les répondants à $\mathrm{E} 1$ étant peut-être plus attirés par une jouabilité (gameplay) complexe, ayant un «coût d'entrée » plus conséquent.

\section{Culture estimée et attrait pour l'histoire}

Par ailleurs, les répondants à E1 se déclarent plus cultivés et plus attirés par l'histoire que ceux d'E2, comme le montrent le tableau suivant, et cela quelle que soit la zone géographique concernée. 


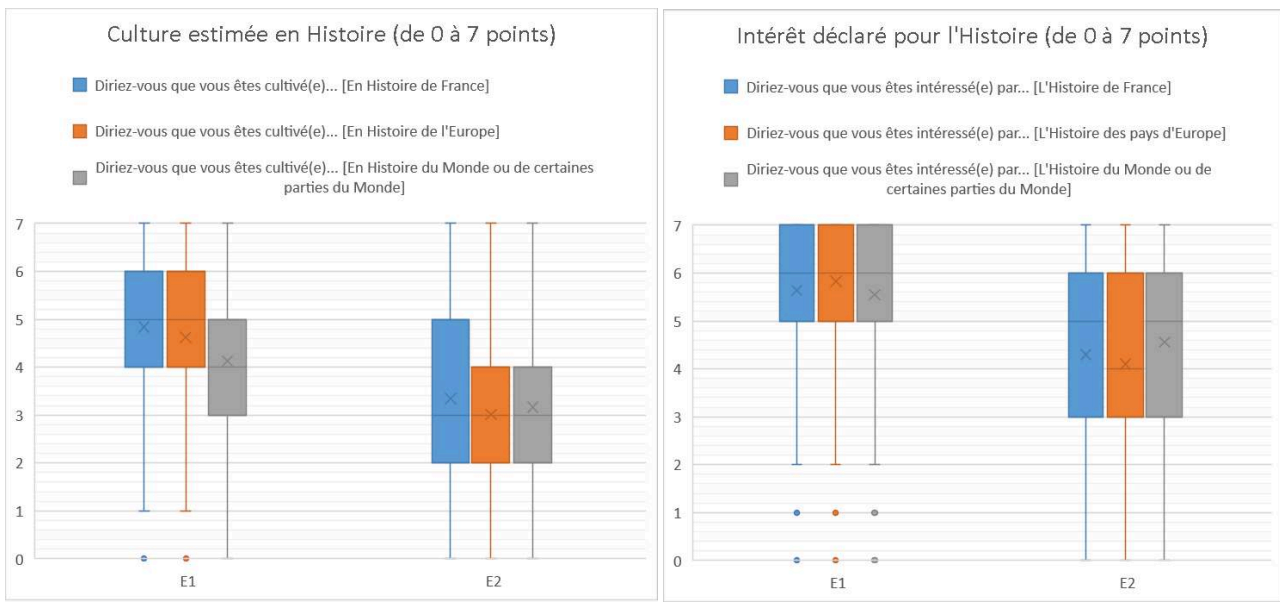

Culture estimée et intérêt déclaré pour l'histoire (sur une échelle de 0 à 7,7 représentant la plus forte culture estimée ou le plus fort intérêt) selon les réponses à E1 (491 réponses) et E2 (323 réponses) sur un diagramme en boîte. Les croix représentent les moyennes, les segments horizontaux les quartiles.

Les valeurs plus fortes pour l'histoire de France peuvent s'expliquer de différentes manières : l'enquête s'adressait à des joueurs francophones, cette histoire est la plus « proche » de nous, la plus abordée à l'école et aussi la plus présente dans les jeux vidéo produits par des occidentaux (ce qui concerne la plupart des jeux cités dans les deux enquêtes). Les zones géographiques les plus «connues" (histoire de France et d'Europe) selon les joueurs entrent en résonance avec des espaces-temps les plus mis en avant dans CK2 et dans EU4 (des extensions ont toutefois mis en avant d'autres zones géographiques hors de l'Europe dans les années suivant la sortie du jeu). Notons l'homogénéité des réponses des répondants E1 (bien plus forte que celles de E2), montrant un public identifiable par son goût pour l'histoire et surtout sa culture estimée dans le domaine. Quant à l'intérêt, il semble avoir une origine plus souvent intrinsèque ou liée à la famille pour E1, alors que les jeux vidéo sont plus souvent un facteur d'intérêt pour E2 : c'est donc le goût pour l'histoire qui semble conduire les joueurs d'E1 vers des jeux historiques. L'école serait alors un facteur d'intérêt, secondaire, mais bien présent, tant pour E1 que pour E2 ; le caractère exploratoire de l'enquête ne nous permet pas de confirmer cette hypothèse (mais les entretiens en cours de traitement permettront de préciser ces analyses).

Par ailleurs, des tests d'indépendance khi-2 montrent que les répondants ayant un fort intérêt pour l'histoire (valeur supérieure à 4,5/7: $77 \%$ d'E1 et $44 \%$ d'E2) sont essentiellement des joueurs de jeux de stratégie et/ou de gestion ${ }^{20}$, ayant un niveau d'étude souvent supérieure ou égal à bac $+2^{21}$ et ayant suivi une filière générale au bac ${ }^{22}$. Aucun lien statistique n'est observé avec le sexe, l'âge et le temps de jeu par mois. En revanche, parmi ceux ayant un score supérieur à 4,5/7 en culture estimée en histoire ( 60 \% d'E1 et $27 \%$ d'E2), la filière au bac n'a pas de lien significatif, ni un niveau d'étude supérieure au bac. Seul le fait de jouer à des jeux de stratégie est corrélé.

\section{Des jeux supports d'informations et source de savoirs}

Si l'on reprend les questions du quizz ( $c f$. tableau 1), et bien que les questions choisies ne montrent que très partiellement ce que l'on peut découvrir dans le $\mathrm{EU}$ ou $\mathrm{CK}$, on 
note : 1) que les répondants à $\mathrm{E} 1$ répondent plus souvent juste (ce qui semble montrer que le jeu est un vecteur de savoirs, transférables dans ces questions) et 2) que les questions qui sont rarement ou non abordées dans les jeux ne sont pas forcément erronées (particulièrement dans E1). Le fait d'avoir vu durant sa scolarité (en particulier pour Q1, Q2, Q4 et Q7) un élément permettant de répondre correctement aux questions ne joue que partiellement (en témoignent les valeurs d'E2). Le tableau suivant montre les fréquences de réponses correctes (trois autres possibilités existent : erreur, ne se prononce pas, le répondant coche une case indiquant qu'il ne connaît pas la réponse, non-répons ${ }^{23}$ ) et l'écart entre E2 et E1, toujours positif (en faveur d'E1) :

Tableau 7

\begin{tabular}{|l|l|l|l|}
\hline Qn & E1 (en \%) & E2 (en \%) & Ecart (E1 - E2) \\
\hline Q1 & 96 & 42 & 54 \\
\hline Q2 & 82 & 67 & 15 \\
\hline Q3 & 89 & 17 & 72 \\
\hline Q4 & 45 & 22 & 23 \\
\hline Q5 & 83 & 21 & 62 \\
\hline Q6 & 74 & 12 & 62 \\
\hline Q7 & 81 & 64 & 17 \\
\hline
\end{tabular}

Fréquence de bonnes réponses au quizz ; la troisième colonne indique l'écart entre E2 et E1. (E1 $=480$ réponses; $E 2=320$ réponses). Rappelons qu'un quart des répondants d'E2 (dont les réponses n'ont pas pu être exploitées) ont quitté le questionnaire lorsqu'ils sont arrivés sur ce quizz. Les pourcentages sont potentiellement plus élevés qu'ils ne l'auraient été.

Les erreurs fréquentes chez $\mathrm{E} 1$ révèlent les confusions entre les informations vues dans CK2 ou EU4, comme par exemple confondre l'Empire Romain d'Orient (ou Byzantin) et l'Empire Ottoman dans la Q2 (alors qu'une date était indiquée sur la carte montrée). En revanche, le fort taux de réussite à la $\mathrm{Q} 7$ manifeste d'une tendance à avoir une plus grande culture historique (dans ce cas précis, sur le premier Empire où les joueurs devaient deviner une date approximative d'une carte, sur laquelle les départements français étaient dessinés). Les étudiants en histoire font peu d'erreurs (ceux-ci représentent une très faible part de l'échantillon : $14 \%$ parmi les étudiants et $5 \%$ de l'échantillon total). Un lien statistique (test de Fisher) existe avec l'âge moyen, mais reste léger : les plus jeunes répondent moins souvent aux questions; ces derniers sont également plus représentés dans E2.

Certains joueurs ont également cité directement (dans le champ texte libre au sein de l'enquête) ce qu'ils avaient appris dans EU4 ou CK2 ; les réponses indiquées dépendent clairement du jeu auquel ils ont joué. Ainsi, parmi les joueurs pratiquant exclusivement $\mathrm{CK} 2$, l'un nous indique : " Je connaissais Charlemagne (comme tout le monde), j'ignorais qu'il avait un frère. Ou que les Norvégiens avaient tenté de prendre l'Angleterre en même temps que Guillaume [...]» $\left(\mathrm{R} 19^{24}\right)$. Un autre écrit avoir appris «le 
fonctionnement de la féodalité »(R418). Chez les joueurs d'EU4, nous trouvons par exemple :

Un élément qui me paraît important de connaître, et que j'ai appris dans Europa Universalis IV, est la Guerre de 30 ans (guerre de religion entre protestant et catholique) car cela permet de comprendre le contexte de l'époque et de comprendre certaines décisions [...] plus tard dans l'histoire. (R50)

Ensuite, lorsque nous demandons où ils ont appris certaines informations (choisies arbitrairement également, souhaitant notamment éviter des concepts complexes à évaluer), la place des jeux vidéo est souvent située devant celle de l'école ou de l'université (particulièrement pour E1, les jeux EU4 et CK2 étant souvent leur source première). Le tableau suivant ne présente qu'une partie des réponses possibles (les deux les plus fréquemment choisies).

Tableau 8

\begin{tabular}{|c|c|c|c|c|}
\hline \multirow[t]{2}{*}{ Fait } & \multicolumn{2}{|c|}{$\begin{array}{l}\text { Dans un jeu } \\
\text { vidéo (en \%) }\end{array}$} & \multicolumn{2}{|c|}{$\begin{array}{l}\text { À l'école ou } \\
\text { l'université } \\
\text { (en \%) }\end{array}$} \\
\hline & E1 & E2 & E1 & E2 \\
\hline Le nom de « Saint Empire Romain Germanique » & 44 & 27 & 33 & 31 \\
\hline $\begin{array}{l}\text { L'électeur du Saint Empire Romain Germanique par des princes } \\
\text { électeurs }\end{array}$ & 63 & 12 & 12 & 11 \\
\hline $\begin{array}{l}\text { L'union ibérique (regroupant les royaumes de Castille et } \\
\text { d'Aragon) }\end{array}$ & 56 & 15 & 19 & 25 \\
\hline L'édit de Nantes & 3 & 1 & 81 & 68 \\
\hline $\begin{array}{l}\text { L'expansion de l'Empire Ottoman durant le Moyen-Âge et la } \\
\text { Renaissance }\end{array}$ & 38 & 22 & 32 & 45 \\
\hline $\begin{array}{l}\text { Le fait que la Papauté disposait d'autres territoires que le Vatican } \\
\text { actuel }\end{array}$ & 41 & 17 & 24 & 30 \\
\hline $\begin{array}{l}\text { La France ne possédant pas ou plus, en } 1444 \text {, la Bretagne, la } \\
\text { Provence, l'Anjou, la Bourgogne, la Normandie et une partie de } \\
\text { l'Aquitaine }\end{array}$ & 44 & 10 & 27 & 26 \\
\hline L'Écosse et l'Angleterre, initialement séparées & 14 & 11 & 36 & 33 \\
\hline La prise de Constantinople en 1453 & 19 & 12 & 60 & 57 \\
\hline
\end{tabular}

Source de certains apprentissages (centrés sur les jeux vidéo ou l'école et l'université). Les autres possibilités étaient : «Un reportage, un livre, Internet », « un film, une série », « autre », " je connais, mais je ne sais plus d'où » et « je ne connaissais pas ». Une seule réponse possible. N =814.

41 Les disparités entre E1 et E2 nous montrent, ici encore, des apprentissages propres aux jeux EU4 et CK2. Certains apprentissages scolaires restent ancrés dans la mémoire, comme l'édit de Nantes ou la prise de Constantinople (marquant pour beaucoup 
d'historiens le début de la Renaissance). Les jeux vidéo (en particulier ceux étudiés) peuvent être un complément aux apprentissages scolaires, comme nous l'ont indiqué des répondants :

Étant étudiant en licence d'allemand, j'ai la chance d'avoir une longueur d'avance sur l'histoire des pays germaniques, contrairement aux autres. Une preuve supplémentaire du manque de connaissance qui peut être comblé par ce type de jeux. (R91)

Je ne savais rien sur l'histoire du SERG (Saint Empire Romain Germanique), ni de l'Asie / Afrique avant la colonisation et je considère qu'une base minime devrait se trouver dans les manuels scolaires. (R50) ${ }^{25}$

Le format graphique peut également être un atout pour mieux comprendre l'histoire dans une approche spatio-temporelle:

J'ai beaucoup appris de la lecture des cartes de départ de CK2 aux différentes dates possibles. Le Moyen Age est rarement abordé du point de vue des cartes (dans mes études en tout cas), et j'ignorais par exemple que les musulmans contrôlaient presque toute l'espagne au sème siècle. Pour moi l'étude des cartes et des évolutions de celles-ci durant toute la période du Moyen Age permet de mieux comprendre certaines situations actuelles, et de mieux comprendre la construction des nations. Je pense que c'est la chose la plus importante, car j'ai beaucoup moins de peine à trouver de nombreux pays maintenant (je suis plutôt mauvais en géographie). (R110).

Plus généralement, un grand nombre de joueurs d'EU4 ou CK2 estiment avoir appris en histoire grâce à ces jeux. Sur une échelle de 0 à 7 , les joueurs estiment avoir appris en histoire grâce à EU4 ou CK2 à hauteur de 5,11 / 7 en moyenne (écart-type de 1,70). En revanche, l'apprentissage effectué n'est pas toujours transféré au niveau des savoirs connus : s'ils sont nombreux à estimer apprendre avec EU4 ou CK2, ils sont moins nombreux à indiquer avoir mieux compris certains événements historiques (moyenne = 4,68 / 7 et écart-type $=1,86$ ) et à faire un lien avec le monde actuel (moyenne $=3,72$ / 7 et écart-type $=2,18$ ). Ainsi, l'information est transmise et constitue bien un savoir disciplinaire, mais ce savoir reste "figé " pour nombre d'entre eux, sans faire lien avec d'autres savoirs qui aideraient à mieux comprendre le monde contemporain (élément également discuté en ce qui concerne les savoirs transmis à l'école par exemple par Perrenoud, 1997). Toutefois, ceci reste délicat à interpréter, l'enquête montrant une " photographie » à un instant précis et ne rendant pas compte des liens que les joueurs ont pu faire plusieurs mois ou années après l'acquisition du savoir. De plus, les jeux concernent une période lointaine de la nôtre, et les méconnaissances des périodes entre les deux peuvent également réduire ce transfert.

\section{Des jeux sources « d'apprentissages tangentiels »}

Si l'apprentissage via certains jeux vidéo ne fait aucun doute parmi les enquêtés d'E1, comme nous venons de le voir, il nous a semblé intéressant de voir si certains joueurs allaient plus loin dans leur apprentissage, reprenant alors le concept d'apprentissage tangentiel, notamment énoncé par Mozelius et al. (2017). Globalement, les joueurs d'EU4 ou de CK2 (E1) vont chercher plus d'information:

Tableau 9

\begin{tabular}{|l|l|}
\hline Les joueurs font-ils des recherches complémentaires ? & Fréquence (en \%) \\
\hline
\end{tabular} 


\begin{tabular}{|l|l|}
\hline Jamais & 5 \\
\hline Cela m'est arrivé 1 fois & 2 \\
\hline Cela m'est arrivé 2 ou 3 fois & 19 \\
\hline Cela m'est arrivé entre 4 et 10 fois & 22 \\
\hline Cela m'est arrivé plus de 10 fois & 52 \\
\hline TOTAL & 100 \\
\hline
\end{tabular}

Les joueurs d'EU4 et CK2 vont-ils chercher plus d'informations après une découverte dans le jeu? $\mathrm{N}=491$.

Il y a une corrélation (test khi-2) forte avec ceux ayant les deux résultats les plus élevés (c'est-à-dire plus de 4 fois) et le fait qu'ils sont surtout joueurs de jeux de stratégie ${ }^{26}$, ont un fort temps de jeu sur EU4 ou CK2 $2^{27}$, sont plus intéressés par l'histoire ${ }^{28}$, et un lien avec la filière au BAC (plus souvent générale) ${ }^{29}$; il n'y a aucun lien statistique noté avec le sexe, l'âge ou le temps passé à jouer.

Nous leur avons également demandé s'ils étendaient leurs recherches :

Tableau 10

\begin{tabular}{|l|l|}
\hline Les joueurs étendent-ils leurs recherches ? À quel point? & $\begin{array}{l}\text { Fréquence } \\
\text { (en \%) }\end{array}$ \\
\hline Très peu : quelques lignes ou une courte vidéo & 9 \\
\hline $\begin{array}{l}\text { Moyennement : quelques pages d'un livre, sur Internet ou une vidéo d'au moins 5 } \\
\text { minutes }\end{array}$ & 50 \\
\hline $\begin{array}{l}\text { Beaucoup : je lis une partie d'un livre et/ou de nombreuses pages sur Internet et/ } \\
\text { ou je regarde au moins 1 reportage sur le sujet }\end{array}$ & 30 \\
\hline $\begin{array}{l}\text { Enormément: plusieurs reportages et/ou un livre entier et/ou plusieurs } \\
\text { encyclopédies et documents entièrement consultés }\end{array}$ & 8 \\
\hline Autre & 3 \\
\hline TOTAL & 100 \\
\hline
\end{tabular}

Les joueurs d'EU4 et CK2 étendent-ils leurs recherches, et à quel point ? N =490.

Les joueurs indiquent aller fréquemment chercher plus d'informations et être prêts à y passer un peu de temps, voire à faire de nombreuses recherches pour combler leur besoin d'information, c'est-à-dire " une sensation qui porterait l'individu à s'engager dans une activité de recherche d'information» (Simonnot, 2007). Pour certains, ce complément concerne d'autres régions du monde et/ou est un moyen de révision ou 
d'approfondissement de leurs connaissances, poussant à aller chercher plus d'informations, notamment dans des ouvrages :

En général, je maitrise bien les événements historiques en Europe grâce à mes précédentes études en Histoire. J'ai de grosses lacunes en histoire hors Europe et les parties avec ce type de pays me pousse souvent à me documenter (d'abord sommairement via Wikipédia, puis plus sérieusement via ma bibliothèque universitaire). J'ai donc progressé en connaissance de l'histoire de l'Inde [...], de l'Afrique [...] et de l'Asie [...]. Le jeu m'a toutefois poussé à réviser beaucoup l'histoire européenne [...], même si ce n'était pas une découverte. (R141)

Pour d'autres, ce sont des abonnements à des chaînes YouTube ou autres communautés qui sont privilégiés pour approfondir leurs connaissances :

Ces jeux ont développé un intérêt pour l'Histoire chez moi, et sans ces jeux je ne serai pas abonné à des chaînes Youtube comme Nota Bene, Herodot'com, Histoire de France et j'en passe. J'ai même découvert l'existence de la République des DeuxNations à mes débuts, alors que maintenant je connais son Histoire, l'évolution de ses frontières, le sauvetage de la ville de Vienne par Jean III Sobieski en 1683, les partitions, etc. [...] (R26)

\section{Conclusions et pistes de recherche}

47 Ainsi, les joueurs d'EU4 et CK2 multiplient les espaces-temps d'apprentissage : certains sont formels (à l'école, l'université, au musée, etc.), d'autres informels (dans les jeux vidéo, etc.). Dans ce contexte, le jeu vidéo est un médiateur de savoirs et d'intérêt pour en apprendre plus. Jouer à des jeux vidéo qui incluent dans leurs contenus des savoirs relève donc, d'une certaine manière, d'une pratique d'information (Gardiès et al., 2010) puisque ladite pratique du jeu vidéo n'a pas de prime abord pour intention la recherche d'information. Et les apprentissages qui en résultent relèveraient alors plutôt de la sérendipité, mais peuvent générer un besoin d'information, ou du moins le stimuler, si ceux-ci sont jugés insuffisants : il faut avoir un intérêt, être motivé pour s'engager dans l'effort ensuite (Simonnot, 2007). De plus, le jeu seul ne suffit pas: si l'on prend l'exemple d'EU4 et de CK2, non seulement les infidélités historiques sont nombreuses une fois la partie débutée, mais nous avons également vu que les joueurs n'arrivaient pas systématiquement à mieux comprendre le monde actuel en en apprenant sur celui d'hier. La nécessité d'un médiateur pour faire le lien entre le reçu via le jeu et la réalité est alors questionnée. Les résultats des recherches d'informations complémentaires ou les échanges sur les forums sur ces jeux (parfois purement historiques) peuvent participer à cette médiation.

Nous pouvons émettre l'hypothèse qu'il y aurait trois niveaux d'apprentissage dans la pratique du jeu vidéo, avec, potentiellement, appropriation de savoirs :

1. Le jeu vidéo comme médiateur de savoirs. À ce niveau, il constitue principalement un support, un matériau d'illustration, et est lui-même médiateur de l'information. Par exemple, on repère l'emplacement des pays, les différentes cultures, les religions, etc. Ceci, nous avons pu le voir dans les analyses: les résultats au quizz entre E1 et E2 et dans les citations d'éléments appris par les joueurs.

2. Le jeu vidéo comme support d'analyse et de compréhension de concepts ou de situations plus ou moins complexes. Si les savoirs et la jouabilité sont fortement imbriqués, une pratique approfondie du jeu (passant notamment par une grande connaissance des mécaniques du jeu) peut permettre d'accéder à des savoirs plus complexes. Par exemple, on peut comprendre les grands principes de la féodalité, des mariages royaux, etc. 
3. Le jeu vidéo comme support de développement de l'esprit critique, notamment en étant capable d'identifier les erreurs du jeu (exemple: infidélités historiques) ou les éléments sujets à controverse. Un des joueurs écrit ainsi que la pratique de ces jeux «permet de prendre ses distances par rapport à ce qu'on peut entendre dans les médias traditionnels, de se faire son propre point de vue sur un événement sans filtre idéologique » (R91), ou encore «ces jeux donnent une autre vision du monde que celle reçu par la société en général » (R114). Krathwohl, 2002), le premier correspondant à la connaissance factuelle, le second à la connaissance conceptuelle (jusqu'à être capable de transférer les savoirs acquis pour mieux comprendre le monde), et le troisième aux derniers niveaux de connaissances énoncés par Krathwohl (connaissances procédurales voire métacognitives), bien que la frontière entre les niveaux soit particulièrement poreuse. Parallèlement à ces niveaux, le jeu vidéo susciterait de l'intérêt pour certaines disciplines (exemples: histoire, géographie, sciences expérimentales), et les recherches menées pour combler un besoin d'information viendraient compléter les manques dans les trois niveaux précédemment énoncés.

Nous souhaitons conforter ces premiers résultats en poursuivant nos recherches avec, d'une part, une étude narrative des jeux pour identifier l'articulation entre savoirs et jouabilité, et, d'autre part, l'analyse d'entretiens (auprès de joueurs des deux jeux étudiés), dans lesquels nous abordons les apprentissages notionnels (comme la féodalité) plutôt que des faits historiques précis, le format de l'entretien s'y prêtant plus. Sont également abordés leur pratique des jeux vidéo et l'impact que cela a eu sur leur goût pour l'histoire-géographie et leurs pratiques informationnelles. Par ailleurs, établir une échelle permettant de classer les jeux vidéo selon leur "profondeur historique » et la fiabilité de leur information reste une autre piste à explorer.

\section{BIBLIOGRAPHIE}

AMATO, É. (2012), « Communication ludique. Origine et puissance d'un nouveau média », Hermès, la revue, 62, 21-26.

AMATO, É. (2014), « Le jeu vidéo, premier média cybernétique de masse », Hermès, la revue, 70, 163-165.

BALMISSE, G. (2002), Gestion de connaissances : Outils et applications du knowledge management, Paris, Eyrolles.

BERRY, V. (2006), «Immersion dans un monde virtuel : jeux vidéo, communautés et apprentissage ", Observatoire des mondes numériques en sciences humaines. Disponible à http:// www.omnsh.org/ressources/557/immersions-dans-un-monde-virtuel-jeux-video-communauteset-apprentissages, consulté le 01/01/2020.

BERRY, V. (2011), «Jouer pour apprendre : est-ce bien sérieux? Réflexions théoriques sur les relations jeu (vidéo) et apprentissage », La revue canadienne de l'apprentissage et de la technologie [En 
ligne], 37(2). Disponible à : https://www.cjlt.ca/index.php/cjlt/article/view/26359, consulté le $01 / 01 / 2020$.

BELISLE, C., BIANCHI, J. et JOURDAN, R. (1999), Pratiques médiatiques. 50 mots-clés, Paris, CNRS Editions.

BORZAKIAN, M. (2013), « Les jeux : quelle définition par ou pour les sciences sociales ? », Loisir et Société, 35, 341-360.

BROUGERE G. (1995), Jeu et éducation, Paris, L'Harmattan.

BROUGERE, G. (2003), « Le jeu : entre éducation et divertissement », Médiation \& Information, 18, $43-52$.

BROUGERE, G. (2005), Jouer/Apprendre, Paris, Economica.

BROUGERE, G. (2019), « Comment penser les relations entre jeu et éducation », Balises : le magasine de la Bpi [En ligne]. Disponible à : https://balises.bpi.fr/education/peut-on-apprendre-en-jouant, consulté le 14/10/2019.

BRUNER, J. (1987), Comment les enfants apprennent à parler, Paris, Retz.

CAILLOIS R. (1967), Les jeux et les hommes : le masque et le vertige, Paris, Gallimard.

CALVERT, S. L. (2005), « Cognitive effects of video games », in Raessens J. \& J. Goldstein (dir.), Handbook of Computer Game Studies, Cambridge, MIT Press, 125-131.

COAVOUX, S., BOUTET, M., ZABBAN, V. (2016). « What We Know About Games. A Scientometric Approach to Game Studies in the 2000s ». Games and Culture, SAGE Publications.

DACHEUX, E. (2009). «La bande dessinée : art reconnu, média méconnu : introduction générale », Hermès, la revue, 54, 11-17.

DARRAS, B. (2003), « Entretien avec André Giordan : apprendre et jouer », MEI, 18, 15-40.

DURIS, O. (2017). « Du jeu au jeu vidéo. Sur l'intérêt des univers vidéo ludiques dans la clinique de la psychose infantile ». Revue de l'enfance et de l'adolescence, 95, 85-98.

GARDIES, C., FABRE, I. et COUZINET, V. (2010), « Re-questionner les pratiques

informationnelles ", Études de communication, 35, 121-132.

GEE, J. P. (2003), « What Video Games Have to Teach us About Learning and Literacy », Computers in Entertainment, 1, 20-20.

GENVO, S. (2011), « Jeux vidéo », Communications, 88, 93-101.

JUUL, J. (2011), Half-Real - Video Games between Real Rules and Fictional Worlds, Cambridge, MIT Press.

KARSENTI, T., BUGMANN, J., PARENT, S. (2019). Apprendre l'histoire avec le jeu vidéo Assassin's Creed ? Une étude exploratoire auprès de 329 élèves. Montréal : CRIFPE.

KLINE, S., DYER-WITHEFORD, N., DE PEUTER, G. (2003), Digital Play - The interaction of Technology, Culture, and Marketing, Montreal \& Kingston, MC Gill-Queen's University Press.

KOEBEL, G. (2018), « Simulating the ages of man : periodization in Civilization V and Europa Universalis IV », The Journal of the Canadian Game Studies Association, 17, 60-76.

KRATHWOHL, D. R., (2002), « A revision of Bloom's Taxonomy : An Overview », Theory into practice, $4,212-218$.

HUIZINGA, (1951), Homo ludens : essai sur la fonction sociale du jeu, Paris, Gallimard. 
JEANNERET, Y. (2000), Y a-t-il vraiment des technologies de l'information?, Villeneuve d'Ascq, Presses universitaires du Septentrion.

LAFRANCE, J.-P., OLIVERI, N., DACHEUX, E. (2012). « Introduction », Hermès, la revue, 62, pp. 9-12.

MEDIAMETRIE (2019), « Le jeu vidéo s'ancre dans le quotidien », Médiamétrie. Disponible à :

https://www.mediametrie.fr/fr/le-jeu-video-sancre-dans-le-quotidien, consultée le 20/01/2020.

MINASSIAN, H. T. et RUFAT, S. (2008), « Et si les jeux vidéo servaient à comprendre la

géographie? », Cybergeo : European Journal of Geography [En ligne], document 418. Disponible à :

https://journals.openedition.org/cybergeo/17502, consulté le 01/02/2020.

MOZELIUS, P., FAGERSTRÖM, A. et SÖDERQUIST, M. (2017), « Motivating factors and tangential learning for knowledge acquisition in educational games », The electronical journal of e-learning, 4 , 343-354.

PERAYA, D. (1999), « Médiation et médiatisation : Le campus virtuel », Hermès, la revue, 25, 153-168.

PERRENOUD, P. (1997) «Vers des pratiques pédagogiques favorisant le transfert des acquis scolaires hors de l'école », 3, 5-16.

PERRIAULT, J. (2012). « Jeux virtuels. Aspects sociocognitifs et sémiotiques », Hermès, la revue, 62 , 92-100.

POINSIGNON, P. (2018). « Jeu vidéo, culture et industrie », Annales des Mines - Gérer et comprendre, 2, 75-76.

SCHAFER, V. et THIERRY, B. (2012), « Le temps des empires. Une partie à jouer pour les historiens », Hermès, la revue, 62, 27-31.

SIMONNOT, B. (2007). «Le besoin d'information : principes et compétences ». Actes de Thémat'IC 2006. Information : besoins et usages, Strasbourg : IUT Robert Schuman, mars 2006. 39-54.

SUDNOW, D. (1983), Pilgrim in the Microworld, New York, Warner Books.

SUTTON-SMITH, B. (1997), The Ambiguity of play, Cambridge, Havard University Press.

TROCMÉ-FABRE, H. (1999), Réinventer le métier d'apprendre : le seul métier durable aujourd'hui, Paris, Eyrolles.

WENGER, E. (1998), Communities of Practice : Learning, Meaning, and Identity, Cambridge University Press, New Ed.

YONNET P. (2004), Huit Leçons sur le sport, Paris, Gallimard.

ZABBAN, V. (2012), « Retour sur les game studies. Comprendre et dépasser les approches formelles et culturelles du jeu vidéo », Réseaux, 173, 137-176.

\section{NOTES}

1. Ce titre est une citation du titre de l'article de Berry (2011).

2. Le réseau LUDUS, créé en 1998 (http://www.lepetitjournaldesprofs.com/reseauludus/nosjeux/), CANOPE (https://http://www.reseau-canope.fr/apprendre-par-le-jeu.html ou encore Eduscol (https://eduscol.education.fr/jeu-numerique/rubrique/1776 ou https:// eduscol.education.fr/numerique/tout-le-numerique/veille-education-numerique/fevrier-2019/ jeux-video-et-education) proposent des contenus à destination des enseignants s'appuyant sur des jeux vidéo 
3. Downloadable content (contenu téléchargeable), payant ou gratuit; il s'agit d'extensions, dont le contenu varie, proposés par l'éditeur.

4. Source : https://store.steampowered.com/stats/?l=french consultée le 23/08/19.

5. Avant la sortie de deux DLC payants, la date de départ était fixée à 1066.

6. Le chiffre peut paraître vertigineux, mais les résultats que nous présentons montrent que ces jeux sont joués pendant plusieurs centaines d'heures (voire plus de 1000 heures), du fait d'une grande rejouabilité et de mises à jour fréquentes. De plus, la complexité des règles peut nécessiter un temps d'apprentissage long.

7. Notamment: Civilization VI (2K Games), Dishonored 2 (Arkane Studio), Call of Duty 4: Modern Warfarare (Infinity Ward), The Witcher 3 (CD Projekt), Fallout 4 (Bethesda Softworks), Skyrim (idem), Age of Empires 2 HD (Ensemble Studios)...

8. Ceci était demandé en plusieurs questions : «à quelle fréquence jouez-vous à des jeux vidéo parmi les catégories suivantes ?» (différentes catégories étaient proposées), "Généralement, combien de temps jouez-vous aux jeux vidéo?» (avec des échelles de valeurs pour plusieurs catégories : en semaine hors vacances, le week-end, pendant les vacances, sur un mois).

9. Des entretiens sont en cours de retranscription et d'analyse et donneront lieu à un article précisant les premiers résultats présentés ici.

10. Les autres possibilités sont: demandeurs d'emploi, autres sans activités professionnelles et autres statuts.

11. Source : CNC, (2014), « Les pratiques de consommation de jeux vidéo des Français », CNC. URL : https://www.cnc.fr/jeu-video/etudes-et-rapports/etudes-prospectives/les-pratiques-deconsommation-de-jeux-video-des-francais_304289, consultée le 22/08/2019.

12. Source: IFOP, (2018), « Les Français et le jeu vidéo », IFOP. URL : https://www.ifop.com/wpcontent/uploads/2018/10/115759-Pr \%C3 \%A9sentation-23.10.2018.pdf, consultée le 22/08/2019. 13. Source : Médiamétrie, (2019), «Le jeu vidéo s'ancre dans le quotidien », Médiamétrie. URL : https://www.mediametrie.fr/fr/le-jeu-video-sancre-dans-le-quotidien consultée le 22/08/2019.

14. Source : https://fr.statista.com/statistiques/481366/comparaison-des-joueurs-de-jeux-videopar-sexe/ consultée le 22/08/2019.

15. Jeux sélectionnés pour l'enquête d'après un classement par les joueurs des jeux vidéo historiques sur Sens Critique: https://www.senscritique.com/top/resultats/ Les_meilleurs_jeux_historiques/465485 (consultée le 19/10/2017).

16. Les développeurs de Sim City ont varié depuis 1989, Maxis ayant toujours été le principal.

17. Développé par Colossal Order \& Tantalus Media, sorti en 2015.

18. Les autres propositions étaient: Action, jeux de tir (FPS), Jeux de rôles, Hack'n'slash, Aventure, et Autre. Les propositions mentionnées dans le tableau étaient : Stratégie (temps réel ou non), Gestion, et Simulation sportive.

19. Ne sont retenus que les Total War de la série basée sur l'Histoire, et non les univers fantastiques.

20. Chi $2=18,93 ; \mathrm{ddl}=8 ; \mathrm{p}=1,52 \%$.

21. Chi2 $=12,53 ; \mathrm{ddl}=4 ; \mathrm{p}=1,38 \%$.

22. Chi $2=10,38 ; \mathrm{ddl}=4 ; \mathrm{p}=3,45 \%$.

23. À ce sujet, notons que les répondants devaient répondre dans un champ de réponse libre.

24. Répondant $n^{\circ} 19$.

25. Les verbatims sont copiés/collés en l'état sans aucune correction de syntaxe ou d'orthographe.

26. Chi $2=17,61 ; \mathrm{ddl}=4 ; \mathrm{p}=0,15 \%$.

27. Chi $2=97,60 ; \mathrm{ddl}=28 ; \mathrm{p}<0,01 \%$.

28. Chi2 $=52,87 ; \mathrm{ddl}=16 ; \mathrm{p}<0,01 \%$.

29. Chi $2=23,92 ; d d l=12 ; p=2,09 \%$. 


\section{RÉSUMÉS}

Nous nous demandons dans cet article en quoi et comment un jeu vidéo peut être source d'apprentissage. Nous proposons d'y répondre en étudiant deux jeux de stratégie/gestion (Crusader Kings 2 et Europa Universalis 4) comme médiateurs de savoirs en histoire. Nous avons procédé à deux enquêtes, une auprès de joueurs de ces jeux et une autre auprès d'individus n'y jouant pas (majoritairement joueurs d'autres jeux), pour un total de 1060 réponses. Il en résulte que les jeux vidéo sont non seulement des porteurs d'informations pouvant devenir des savoirs pour les joueurs, mais qu'ils stimulent une curiosité menant à des pratiques d'informations plurielles, permettant d'acquérir d'autres savoirs, ce qui relève de l'apprentissage tangentiel.

In this article, our question is to know how a video game can be a source of learning? We will study two strategy / management games (Crusader Kings 2 and Europa Universalis 4) as mediators of knowledge in History. We made two surveys, one among players of these games and another among people who don't play them (mainly players of other video games), for a total of 1060 answers. As a result, video games are supports of information which could become knowledge, and also a motivation for plural practices of information, allowing to acquire other knowledge which falls under tangential learning.

\section{INDEX}

Mots-clés : jeu vidéo, médiation des savoirs, apprentissage tangentiel, pratiques d'information Keywords : video game, knowledge mediation, tangential learning, practices of information

\section{AUTEUR}

CLÉMENT DUSSARPS

Laboratoire IMS CNRS UMR 5218 - Equipe RUDII. Université de Bordeaux. 